\title{
Ludificación y sus posibilidades en el entorno de blended learning: revisión documental
}

\section{Gamification and its possibilities in the blended learning environment: literature review}

\author{
Ángel Torres-Toukoumidis \\ Universidad Politécnica Salesiana, UPS (Ecuador) \\ Luis Miguel Romero Rodríguez \\ Universidad Internacional de la Rioja, UNIR (España) \\ Amor Pérez Rodríguez \\ Universidad de Huelva, UHU (España)
}

\section{Resumen}

La ludificación en el entorno de aprendizaje semipresencial constituye una innovación de alto impacto en las posibilidades de aprendizaje. Se considera que comparte aspectos con el blended learning, en relación con el aumento de la motivación y la optimización en la obtención de resultados respecto a una actividad para estimular el aprendizaje. En este sentido, este trabajo plantea un análisis de publicaciones en bases de datos internacionales en las que se aborda la cuestión de la ludificación en el entorno de blended learning. Para ello se realiza una revisión de literatura científica, aplicando la documentación como método para la sistematización. El estudio que se presenta analiza 34 aportaciones entre 2012 y 2017* indexadas en las bases de datos WOS y Scopus, utilizando una estrategia de búsqueda basada en cuatro criterios: público (destinatario), tópico, diseño metodológico y conclusiones principales. Los resultados demuestran que las publicaciones reseñadas se centran en estudiantes de educación superior, especialmente del ámbito de la ingeniería, exponen prototipos y modelos, y presentan diseños metodológicos en los que predomina el marco teórico-conceptual, seguido de estudios empírico cuantitativos. Se deduce, en consecuencia, entre otros aspectos, la necesidad de seguir impulsando la convergencia ente ludificación y blended learning, dado el efecto positivo que conlleva en relación con la motivación, retroalimentación y en la adquisición de competencias por parte de los estudiantes.

Palabras clave: enseñanza y formación; educación tecnológica; juego educativo; aprendizaje; interacción; motivación. 


\begin{abstract}
The use of gamification in the blended learning environment constitutes an innovation that greatly impacts learning possibilities. It shares features with blended learning, such as an increase in motivation and the optimization of results with respect to an activity that stimulates learning. In this sense, this paper outlines an analysis of publications in international databases in which the subject of gamification is addressed within the blended learning environment. This scientific literature review has been prepared using documentation as a method of systematization. This research analyzes 34 contributions between 2012 and $2017^{*}$ indexed in the WOS and Scopus databases, using a search strategy with four criteria: recipients, topic, methodological design and main conclusions. Results demonstrate that the documented publications are focused on higher education students, especially in the area of engineering, display prototypes and models and feature methodological designs, in which the conceptualtheoretical framework predominates, followed by empirical and quantitative research. Amongst other outcomes, this paper concludes that there is a need to continue boosting the convergence between gamification and blended learning, due to its positive effects related to motivation, feedback and the acquisition of competencies by students.
\end{abstract}

Keywords: teaching and training; technological education; educational game; learning; learning strategy; interaction; motivation.

La aparición de las innovaciones tecnológicas en el último medio siglo ha tenido un enorme impacto en las posibilidades de aprendizaje, especialmente con la convergencia entre dos prácticas innovadoras, el aprendizaje semipresencial o blended learning y la ludificación o gamification. La Comisión Europea (2013) aboga por la presencia de ambas en los nuevos programas de Eramus+ y Horizon 2020, en los que el blended learning constituye una oportunidad para utilizar recursos educativos abiertos de calidad, mientras que la ludificación impulsa la conectividad e interoperabilidad.

El blended learning es la integración de la modalidad "en línea” y presencial (Garrison y Kanuka, 2004; Graham, 2013) que conecta la tradición y la innovación con el impacto de las iniciativas políticas del sistema educativo (Moskal, Dziuban y Hartman, 2013), combinando múltiples medios diseñados para promover el aprendizaje y el comportamiento aprendido (Singh, 2003). Si bien Driscoll (2002) lo define como un proceso de transición temporal que encamina progresivamente a los estudiantes de la educación tradicional a la educación a distancia, hoy en día se ha asentado como un formato aceptado que incluye actividades de instrucción y oportunidades de colaboración que realzan el compromiso emocional de los estudiantes con sus compañeros e instructores (Lim, Morris y Kupritz, 2007).

Aunque el blended learning no es una práctica formativa nueva, el efecto positivo generado en la motivación (Rovai y Jordan, 2004; Woltering, Herrler, Spitzer y Spreckelsen, 2009) y en los resultados académicos de los estudiantes (Hung, 2014; Lim y Morris, 2009; López-Pérez, Pérez-López y Rodríguez-Ariza, 2011; Poon, 2013; 
Zacharis, 2015) ha sostenido su continuo desarrollo y adopción por las instituciones educativas (Picciano, Dziuban y Graham, 2013), incorporando recursos instructivos adicionales y elementos que estimulan las interacciones (Means, Toyama, Murphy y Baki, 2013), entre ellos, la ludificación (Barata, Gama, Fonseca y Gonçalves, 2013).

La ludificación es un recurso pedagógico con diseño instructivo (Railean, 2017) enfocado al uso de elementos de diseño de juego en contextos no lúdicos (Deterding, Dixon, Khaled y Nacke, 2011). Específicamente, la ludificación se configura como experiencia inmersiva, que transforma los principios de la educación tradicional en nuevos parámetros de aprendizaje fundamentados en la motivación (Caponetto, Earp y Ott, 2014). Un alto nivel de motivación puede determinar que una persona le otorgue significado al hecho de completar una tarea (Muntean, 2011). Para ello es necesario reconocer la diferenciación entre motivación extrínseca y motivación intrínseca. Según Ryan y Deci (2000) la motivación extrínseca lleva a realizar una actividad únicamente por su valor instrumental para cumplir objetivos, mientras que la motivación intrínseca es la satisfacción que deriva de realizar una actividad. Rodgers, Hall, Duncan, Pearson, y Milne (2010) explican que la motivación intrínseca sucede cuando un sujeto hace algo por el simple placer que obtiene al realizarlo, mientras que la extrínseca se da a partir de la obtención de reconocimientos, premios o autorrealización. El estudio empírico realizado por Mekler, Brühlmann, Opwis y Tuch (2014) demuestra que la motivación intrínseca no depende dela implementación de elementos de ludificación que componen la motivación extrínseca, sino en todo caso por factores sociales y contextuales del proceso de ludificación.

Hamari, Koivisto y Sarsa (2014) estiman que patrones incorporados en la ludificación como el aumento de la actividad del usuario, la interacción social o la calidad y la productividad de las acciones, emergen como resultado de la motivación intrínseca de la actividad. Desde esta perspectiva, el compromiso o engagement hacia una actividad con elementos de ludificación, es el resultado de un cambio de comportamiento derivado de la motivación (Hanus y Fox, 2015; Kapp, 2012; Su y Cheng, 2015). Otro aspecto destacable es el efecto en las calificaciones de los estudiantes, en la que Iosup y Epema (2014); Dicheva, Dichev, Agre y Angelova, (2015); Knutas, Ikonen, Nikula, y Porras (2015); y Laskowski (2015) consideran que se exhiben cambios positivos en las puntuaciones de los participantes en todos los niveles del sistema educativo, especialmente en la educación superior.

Independientemente de si la finalidad es enseñar matemáticas (Faghihi, et al., 2014), biología (Drace, 2013), una segunda lengua (Flores, 2015) o medicina (Nevin, et al. 2014), las experiencias que resultan de la ludificación tienen un mismo objetivo, aumentar la motivación y optimizar la obtención de resultados de las personas respecto a una actividad, estimulando el aprendizaje (Landers y Callan, 2011; Simões, Redondo y Vilas, 2013; Kapp, 2012). Por tanto, reconociendo a priori que la ludificación comparte aspectos con el blended learning, este estudio pretende profundizar en la incorporación de la ludificación a dicho entorno. 


\section{METODOLOGíA}

Admitiendo que la combinación entre ludificación y aprendizaje semiprensencial es reciente, y que posee cierto respaldo empírico y descubrimientos parciales (DeMarcos, García-López y García-Cabot, 2016), el planteamiento de este estudio se basa en una revisión de literatura científica, aplicando la documentación como método de recolección (Sampieri, Collado y Lucio, 2014). El objetivo es examinar sistemáticamente la información presentada en bases de datos internacionales sobre la ludificación en el entorno de blended learning. Para lograrlo se identificaron artículos y manuscritos publicados en Web of Science (WOS) y Scopus sobre esta temática, y se organizó la información según un conjunto de criterios determinados por Wollscheid, Sjaastad y Tømte (2016), el cual, se ha utilizado anteriormente en el análisis de literatura científica sobre blended learning.

La delimitación propuesta se apoya en Aghaei Chadegani, Salehi, Yunus, Farhadi, Fooladi, Farhadi y Ale-Ebrahim (2013) que presentan las colecciones de Scopus y Web of Science como las principales bases de datos multidisciplinarias de literatura científica en la actualidad. Complementariamente, la exploración se realiza en artículos de revistas científicas y en actas de conferencias dado que tales tipos de publicaciones aparecen en ambas colecciones y exhiben información suficiente para el análisis.

El estudio se realiza considerando las fechas desde la aparición de cada una de las bases de datos hasta la actualidad (febrero 2017), aunque, siguiendo los parámetros de la estrategia de búsqueda, los resultados en ambas presentan 2012 como fecha del primer artículo indexado. Consecuentemente, el rango estimado es 2012- 2017* (hasta el mes de febrero). La estrategia de búsqueda aplica la opción "tema" en Web of Science (WOS), mientras que en Scopus se utiliza la opción "artículo, título, resumen y palabras clave” combinándose palabras en inglés y español, utilizando el operador booleano AND para conectar los diferentes conceptos. Esto nos permite asociar "gamification" AND "blended learning"; "ludificación" AND "aprendizaje semipresencial"; "ludificación" AND "blended learning"; "gamification" AND "aprendizaje semipresencial". Únicamente se obtienen resultados cuando se emplean los términos en inglés, "gamification" AND "blended learning". 
Gráfico 1. Publicaciones en WOS y Scopus (2012-2017*) sobre "gamification" y "blended learning"

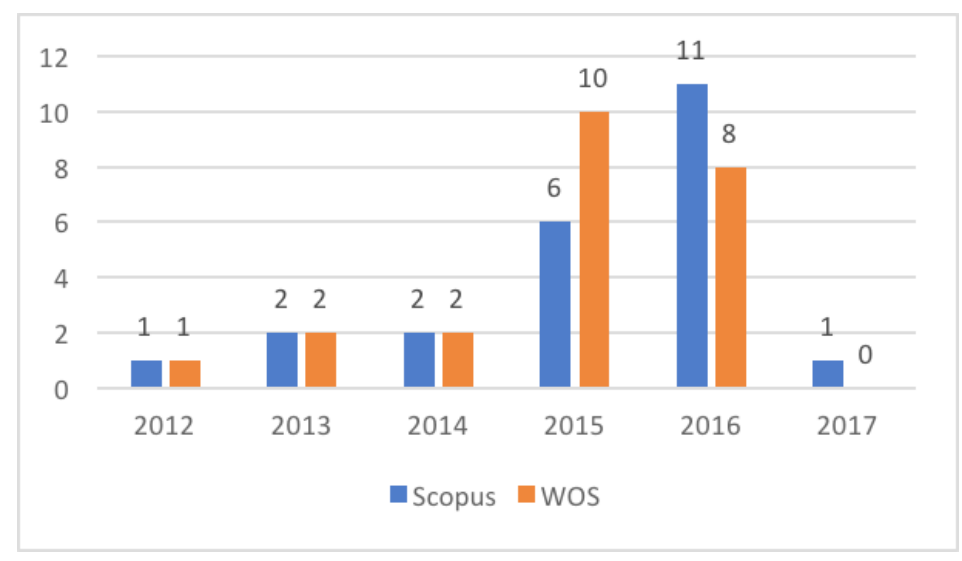

Se consideraron 49 publicaciones, 23 de Web of Science (WOS) y 26 de Scopus, siguiendo la estrategia de búsqueda descrita, de las que 15 publicaciones pertenecientes a revistas científicas y actas de conferencias indexados en ambas bases de datos se encuentran reiteradas (Arnab, Bhakta, Merry, Smith, Star, y Duncan, 2016; Arnab, Tombs, Duncan, Smith, y Star, 2015; Baker, Bujak y DeMillo, 2012; Berkling, 2015; Dagnino, Antonaci, Ott, Lavagnino, Bellotti, Berta, y De Gloria, 2015; Dichev y Dicheva, 2013; Friess, et al., 2015; Hew, Huang, Chu, y Chiu, 2016; Huang, 2016; Lennon, Abbott y McIntosh, 2015; Lückemeyer, 2015; Mozelius, Collin y Olsson, 2015; Priego y Peralta, 2013; Tan y Hew, 2016; Velázquez, 2015). Por lo tanto, la muestra de este estudio ha constado de 34 publicaciones. Por su parte, el hecho de que las publicaciones en conferencias superen a las revistas científicas acontece cuando se trata de estudios en proceso de desarrollo con material preliminar, que se canaliza hacia temas innovadores (Drott, 1995).

El análisis se ha realizado considerando los 4 criterios de Wollscheid, Sjaastad y Tømte (2016) que facilitan la categorización y síntesis de la información recabada sobre la convergencia entre "gamification" y "blended learning" en la Web of Science (WOS) y Scopus:

- Público: educación infantil, educación primaria, educación secundaria, educación superior y formación de formadores.

- Tópico: áreas concomitantes con el estudio.

- Diseño metodológico: teórico-conceptual, empírico con técnicas cualitativas, empírico con técnicas cualitativas, empírico con técnicas cuantitativas y cualitativas.

- Conclusiones principales: efectos y consecuencias de los resultados. 
De esta forma, en relación con el público en el que se centran los estudios analizados, las categorías van desde el primer nivel de enseñanza hasta la formación de expertos, profesores e instructores, permitiendo conocer la etapa educativa en la que se combinan ludificación y aprendizaje semipresencial. El criterio tópico, referido al área temática de cada estudio, se relaciona con las palabras clave y objetivos generales de las investigaciones revisadas (Rivera, 2004). El diseño metodológico sigue la categorización de Martínez-Nicolás y Saperas-Lapiedra (2011. Por último, las conclusiones compendian las aportaciones de las 34 publicaciones que conforman la muestra, omitiendo la estructuración de elementos de ludificación, ya que según Landers (2014) cada implementación de la ludificación se encuentra dentro de un escenario particular que incluye condiciones y variables propias.

\section{RESULTADOS}

\section{Público}

Entre las publicaciones revisadas, el $67 \%$ utiliza en las investigaciones muestras referidas a estudiantes de educación superior, destacándose un 38\% de estudiantes de ingeniería, frente a un $23 \%$ de estudiantes de diversas ramas del conocimiento y un $6 \%$ de estudiantes exclusivamente de ciencias sociales. Por otra parte, un $15 \%$ son publicaciones orientadas a la educación secundaria, un $8 \%$ a educación primaria, un $5 \%$ de carácter general para todo público, y un $3 \%$ se dirige a la formación de formadores.

Tabla 1. Distribución de las publicaciones según el público

\begin{tabular}{|c|c|}
\hline Público & Publicaciones \\
\hline $\begin{array}{l}\text { Estudiantes de } \\
\text { Educación Superior }\end{array}$ & $\begin{array}{l}\text { Bahji, Lefdaoui y El Alami, 2013; Arnab et al., 2015; Bahji, El } \\
\text { Alami y Lefdaoui, 2015; Berkling y Thomas, 2013; Noor, 2013; } \\
\text { Thomas y Berkling, 2013; Berkling y Thomas, 2014; Barros, et al., } \\
\text { 2015; Berkling, 2015a; Berkling, 2015b; Berkling y Zundel, 2015; } \\
\text { Dagnino et al., 2015; Lückemeyer, 2015; Mozelius, Collin y Olsson, } \\
\text { 2015; Pirker, Gutl y Astatke, 2015; Velázquez, 2015; Arnab et al., } \\
\text { 2016; Baksa-Haskó, 2016; Hew et al., 2016; Huang, 2016; Olsson y } \\
\text { Mozelius, 2016; Priego y Peralta, 2016; Tan y Hew, 2016. }\end{array}$ \\
\hline $\begin{array}{l}\text { Estudiantes de } \\
\text { secundaria }\end{array}$ & $\begin{array}{l}\text { Ferzli, Pigford y Black, 2015; Orooji, Taghiyareh y Nasirifard, } \\
\text { 2015; Lennon, Abbott y McIntosh, 2015; Figueiredo, Godejord y } \\
\text { Rodrigues, 2016; Pedersen et al., 2016. }\end{array}$ \\
\hline $\begin{array}{l}\text { Estudiantes de } \\
\text { primaria }\end{array}$ & $\begin{array}{l}\text { Lemus, Baessa y García, 2014; Lemus y Pinto, 2014; Friess, et al., } \\
\text { 2015. }\end{array}$ \\
\hline General (todo público) & Baker, Bujak y DeMillo, 2012; Dichev y Dicheva, 2013. \\
\hline $\begin{array}{l}\text { Formación a } \\
\text { instructores }\end{array}$ & 13. \\
\hline
\end{tabular}




\section{Tópico}

En cuanto a las palabras clave y objetivos generales, el $47 \%$ de artículos tratan de diseño, configuración de prototipos y métodos en los que confluye la ludificación y el aprendizaje semipresencial. Un $29 \%$ se enfocan a la realización de comparativas, resaltando convergencias, diferencias y problematizaciones entre modalidades de enseñanza (15\%), percepciones de los estudiantes (9\%) y elementos de ludificación (5\%). Finalmente, el $23 \%$ se refiere a la profundización de factores particulares entre los que destaca la investigación sobre motivación (12\%) y la enseñanza de asignaturas concretas (11\%).

Tabla 2. Distribución de las publicaciones según el tópico

\begin{tabular}{|l|l|}
\hline \multicolumn{1}{|c|}{ Tópico } & \multicolumn{1}{c|}{ Publicaciones } \\
\hline Diseño, configuración de \\
$\begin{array}{l}\text { prototipos y métodos } \\
\text { y Pinto, 2014; Thomas y Berkling, 2013; Lemus, Baessa y García, } \\
\text { 2014; Arnab, et al., 2015; Lückemeyer, 2015; Pedersen et al., } \\
\text { 2016; Berkling y Zundel, 2015; Dagnino et al., 2015; Ferzli, } \\
\text { Pigford y Black, 2015; Mozelius, Collin y Olsson, 2015; Orooji, } \\
\text { Taghiyareh y Nasirifard, 2015; Velázquez, 2015; Baksa-Haskó, } \\
\text { 2016; Figueiredo, Godejord y Rodrigues, 2016. }\end{array}$ \\
\hline $\begin{array}{l}\text { Comparativas, } \\
\text { convergencias, } \\
\text { diferencias y } \\
\text { problematizaciones }\end{array}$ & $\begin{array}{l}\text { Baker, Bujak y DeMillo, 2012; Berkling y Thomas, 2013; Noor, } \\
\text { 2013; Priego y Peralta, 2013; Berkling y Thomas, 2014; Bahji, El } \\
\text { Alami y Lefdaoui, 2015; Berkling, 2015b; Arnab et al., 2016; Hew } \\
\text { et al., 2016; Tan y Hew, 2016. }\end{array}$ \\
\hline $\begin{array}{l}\text { Profundización de } \\
\text { factores particulares }\end{array}$ & $\begin{array}{l}\text { Barros, et al., 2015; Berkling, 2015a; Friess, et al., 2015; Lennon, } \\
\text { Abbott y McIntosh, 2015; Pirker, Gutl y Astatke, 2015; Huang, } \\
\text { 2016; Olsson y Mozelius, 2016; Priego y Peralta, 2016. }\end{array}$ \\
\hline
\end{tabular}

\section{Diseño metodológico}

En el diseño metodológico, el proceso sistemático más utilizado es la fundamentación a través de un marco teórico-conceptual (38\%). En esta sección se evidencian dos tendencias, la primera orientada al desarrollo teórico-holístico de prototipos de asignaturas y simuladores de entornos blended learning. Su contraparte se refiere a estados de la cuestión, revisiones de literatura sobre herramientas educativas, modos de docencia, casos de experimentación en entorno virtual y convergencias de experiencias. En relación con los estudios empíricos destaca el empleo de técnicas cuantitativas (32\%), frente a las mixtas (21\%) y cualitativas (9\%). Asimismo, los instrumentos utilizados en los estudios empíricos son las encuestas y cuestionarios, en un $35 \%$, y la observación con grupos de control, en un $23 \%$. Por otro lado, el tamaño de las muestras es sumamente variable, esto significa que no existe 
una magnitud común del muestreo en los estudios que combinan la ludificación con el entorno de aprendizaje semipresencial.

Tabla 3. Distribución de las publicaciones según el diseño metodológico

\begin{tabular}{|l|l|}
\hline \multicolumn{1}{|c|}{ Metodología } & \multicolumn{1}{c|}{ Publicaciones } \\
\hline $\begin{array}{l}\text { Marco teórico- } \\
\text { conceptual }\end{array}$ & $\begin{array}{l}\text { Bahji, Lefdaoui y El Alami, 2013; Baker, Bujak y DeMillo, 2012; } \\
\text { Dichev y Dicheva, 2013; Noor, 2013; Arnab, et al., 2015; Berkling } \\
\text { y Zundel, 2015; Dagnino et al., 2015; Ferzli, Pigford y Black, } \\
\text { 2015; Friess, et al., 2015; Lennon, Abbott y McIntosh, 2015; } \\
\text { Lückemeyer, 2015; Pirker, Gutl y Astatke, 2015; Velázquez, 2015. }\end{array}$ \\
\hline $\begin{array}{l}\text { Empírico con técnicas } \\
\text { cuantitativas }\end{array}$ & $\begin{array}{l}\text { Berkling y Thomas, 2013; Priego y Peralta, 2013; Thomas y } \\
\text { Berkling, 2013; Berkling y Thomas, 2014; Barros, et al., 2015; } \\
\text { Berkling, 2015a; Mozelius, Collin y Olsson, 2015; Baksa-Haskó, } \\
\text { 2016; Huang, 2016; Pedersen et al., 2016; Priego y Peralta, 2016. }\end{array}$ \\
\hline $\begin{array}{l}\text { Empírico con técnicas } \\
\text { cuantitativas y } \\
\text { cualitativas }\end{array}$ & $\begin{array}{l}\text { Lemus, Baessa y García, 2014; Lemus y Pinto, 2014; Berkling, } \\
\text { 2015b; Arnab et al., 2016; Hew et al., 2016; Olsson y Mozelius, } \\
\text { 2016; Tan y Hew, 2016. }\end{array}$ \\
\hline $\begin{array}{l}\text { Empírico con técnicas } \\
\text { cualitativas }\end{array}$ & $\begin{array}{l}\text { Bahji, El Alami y Lefdaoui, 2015; Orooji, Taghiyareh y } \\
\text { Nasirifard, 2015; Figueiredo, Godejord y Rodrigues, 2016. }\end{array}$ \\
\hline
\end{tabular}

\section{Conclusiones principales}

El último criterio nos permite valorar las aportaciones de cada una de las investigaciones seleccionadas. La convergencia entre ludificación y aprendizaje semipresencial se presenta como positiva en el $88 \%$ de los textos analizados. Específicamente, un 44\% revela la importancia de la motivación e implicación de los estudiantes en las actividades, impulsando la efectividad, el logro de objetivos, el desarrollo de objetivos prácticos, ejecución de tareas y resolución de problemas. $26 \%$ señala la retroalimentación y el entorno colaborativo estudiante-estudiante, y estudiante-instructor como un patrón común en las plataformas blended-learning, mientras que el $18 \%$ hace hincapié en la mejora de los resultados sobre competencias específicas del programa impartido, entre ellas se enfatiza su aplicación en operaciones matemáticas, lenguaje audiovisual y ciencias computacionales. Otros estudios consideran la práctica de la ludificación de manera menos influyente en el blended learning (12\%), ya que aún amerita mayor desarrollo científico (9\%) o porque simplemente se concibe como un obstáculo para el aprendizaje en la modalidad semipresencial (3\%). 
Tabla 4. Distribución de las publicaciones según las conclusiones principales

\begin{tabular}{|l|l|}
\hline \multicolumn{1}{|c|}{$\begin{array}{c}\text { Conclusiones } \\
\text { principales }\end{array}$} & \multicolumn{1}{c|}{ Publicaciones } \\
\hline Motivación & $\begin{array}{l}\text { Bahji, Lefdaoui y El Alami, 2013; Priego y Peralta, 2013; Lemus } \\
\text { y Pinto, 2014; Thomas y Berkling, 2013; Lemus, Baessa y García, } \\
\text { 2014; Bahji, El Alami y Lefdaoui, 2015; Berkling y Zundel, 2015; } \\
\text { Dagnino et al., 2015; Ferzli, Pigford y Black, 2015; Lennon, } \\
\text { Abbott y McIntosh, 2015; Lückemeyer, 2015; Mozelius, Collin y } \\
\text { Olsson, 2015; Pirker, Gutl y Astatke, 2015; Velázquez, 2015; Hew } \\
\text { et al., 2016. }\end{array}$ \\
\hline Retroalimentación & $\begin{array}{l}\text { Berkling y Thomas, 2014; Barros, et al., 2015; Berkling, 2015b; } \\
\text { Orooji, Taghiyareh y Nasirifard, 2015; Arnab et al., 2016; Baksa- } \\
\text { Haskó, 2016; Olsson y Mozelius, 2016; Priego y Peralta, 2016; } \\
\text { Tan y Hew, 2016. }\end{array}$ \\
\hline Habilidades & $\begin{array}{l}\text { Baker, Bujak y DeMillo, 2012; Dichev y Dicheva, 2013; Arnab et } \\
\text { al., 2015; Friess, et al., 2015; Huang, 2016; Pedersen et al., 2016. }\end{array}$ \\
\hline $\begin{array}{l}\text { Falta mayor desarrollo } \\
\text { científico }\end{array}$ & Berkling y Thomas, 2013; Noor, 2013; Berkling, 2015a. \\
\hline Obstáculo & Figueiredo, Godejord y Rodrigues, 2016. \\
\hline
\end{tabular}

\section{DISCUSIÓN}

Hace más de una década, Garrison y Kanuka (2004) consideraban que la ludificación permitiría potenciar las experiencias de aprendizaje. Hoy en día, dicho supuesto además de concretarse en los sistemas de formación a distancia (De-Marcos, Domínguez, Saenz-de-Navarrete y Pagés, 2014) y presencial (Nicholson, 2012; Hanus y Fox, 2015), también se puede verificar, a través de la revisión documental realizada, que la ludificación actúa como catalizador en los sistemas de formación semipresencial. Se evidencia así la conjunción de las mecánicas de juego (puntos, medallas, niveles, tabla de posiciones, retos, etc.) en el fomento de la motivación, feedback y habilidades específicas de cada ámbito.

En total se revisaron 8 artículos de revistas científicas y 26 actas de conferencias provenientes de Scopus y Web of Science (WOS). Esta revisión refleja el panorama actual de la vinculación entre ludificación y aprendizaje semipresencial. Dicho panorama, en relación con el criterio público, se perfila con estudios (23 publicaciones) en los que la muestra predominante son los estudiantes de educación superior. En cuanto a los tópicos, 16 se refieren al diseño y configuración de prototipos. El criterio del diseño metodológico dominante (en 13 publicaciones) es la fundamentación del marco teórico-conceptual. Finalmente, en lo concerniente a las conclusiones, 30 publicaciones reiteran aquellas referidas a beneficios de esta convergencia, en especial, a la repercusión en la motivación de los participantes. 
Por otro lado, se constata la carencia de publicaciones sobre la formación de formadores, pese a la responsabilidad de orquestar y monitorizar el proceso de aprendizaje semipresencial (Pérez-Sanagustin, Hernández-Leo, Santos, Kloos, y Blat, 2014), así como de trabajos relacionadas con la estructuración de modelos y prototipos con un tratamiento empírico, basándose únicamente en el desarrollo teórico-conceptual. En definitiva, se evidencia la oportunidad de seguir avanzando sobre la naturaleza de esta convergencia, fundamental, como se muestra, en el impulso de la socialización, aprendizaje y motivación de los estudiantes.

\section{REFERENCIAS BIBLIOGRÁFICAS}

Aghaei Chadegani, A., Salehi, H., Yunus, M. M., Farhadi, H., Fooladi, M., Farhadi, M., y Ale Ebrahim, N. (2013). A Comparison between Two Main Academic Literature Collections: Web of Science and Scopus Databases. Asian Social Science, 9(5), 18-26. doi: https://doi.org/10.5539/ass. v9n5p18

Arnab, S., Bhakta, R., Merry, S. K., Smith, M., Star, K., y Duncan, M. (2016). Competition and Collaboration Using a Social And Gamified Online Learning Platform. 1oth European Conference on Games Based Learning: ECGBL 2016 (pp. 19-27). Recuperado de https://goo. gl/AIq1ii

Arnab, S., Tombs, G., Duncan, M., Smith, M., y Star, K. (2015). Towards the Blending of Digital and Physical Learning Contexts with a Gamified and Pervasive Approach. International Conference on Games and Learning Alliance (pp. 452-46o). Springer International Publishing. doi:https://doi. org/10.1007/978-3-319-40216-1 50

Bahji, S. E., Lefdaoui, Y., y El Alami, J. (2013). Enhancing Motivation and Engagement: A Top-Down Approach for the Design of a Learning Experience According to the S2P-LM. iJET, 8(6), 35-41. doi: https:/A doi.org/10.3991/ijet.v8i6.2955

Bahji, S. E., El Alami, J., y Lefdaoui, Y. (2015). Learners' Attitudes Towards ExtendedBlended Learning Experience Based on the S2P Learning Model. International Journal of Advanced Computer Science
\& Applications, 1(6), 70-78. doi: https:/ doi.org/10.14569/ijacsa.2015.061010

Baker, P. M., Bujak, K. R., y DeMillo, R. (2012). The evolving university: Disruptive change and institutional innovation. Procedia Computer Science, 14, 330335. doi: https://doi.org/10.1016/j. procs.2012.10.037

Baksa-Haskó, G. (2016). Efficiency Over 1000 Students-The Evolution of an Online Course: From e-Learning to Flipped Classroom. International Conference on Interactive Collaborative Learning (pp. 237-245). Springer, Cham. doi: https:/ doi.org/10.1007/978-3-319-50340-0 20

Barata, G., Gama, S., Fonseca, M. J., y Gonçalves, D. (2013). Improving student creativity with gamification and virtual worlds. Proceeding First International Conference on Gameful Design, Research, and Applications (pp. 95-98). ACM. doi: https://doi. org/10.1145/2583008.2583010

Barros, M., Moura, A., Borgmann, L., y Terton, U. (2015). Blended Learning in Multi-disciplinary ClassroomsExperiments in a Lecture about Numerical Analysis. Proceedings of the 7 th International Conference on Computer Supported Education (pp. 196-204). SciTePress. doi: https://doi. org/10.5220/0005409001960204

Berkling, K., y Thomas, C. (2013). Gamification of a Software Engineering course and a detailed analysis of the 
factors that lead to it's failure. Interactive Collaborative Learning (ICL), 2013 International Conference (pp. 525-530). IEEE. Recuperado de http://ieeexplore. ieee.org/document/6644642/

Berkling, K., y Thomas, C. (2014). Looking for Usage Patterns in e-Learning Platforms-A Step Towards Adaptive Environments. International Conference on Computer Supported Education (pp. 144-152). doi: https://doi. org/10.5220/0004909001440152

Berkling, K. (2015a). Connecting Peer Reviews with Students' MotivationOnboarding, Motivation and Blended Learning. International Conference on Computer Supported Education (pp. 24-33). Springer International Publishing. doi: https://doi. org/10.5220/0005410200240033

Berkling, K. (2015b). Gamification Behind the Scenes. International Conference on Computer Supported Education (pp. 274292). Springer International Publishing. doi: https://doi.org/10.1007/978-3-31929585-5 16

Berkling, K. M., y Zundel, A. (2015). Change Management: Overcoming the Challenges of Introducing Self-Driven Learning. International Journal of Engineering Pedaqoqu, 5(4), 38-46. doi: https://doi. org/10.3991/ijep.v5i4.4945

Caponetto, I., Earp, J. y Ott, M. (2014). Gamification and Education: A Literature Review. En F. Nah, (Ed.), Business Lecture Notes in Computer Science. (401409). Ginebra: Springer International Publishing. doi: http://dx.doi. org/10.1007/978-3-319-07293-7 39

Comisión Europea. (2013). Apertura de la educación: Docencia y aprendizaje innovadores para todos a través de las nuevas tecnologías y recursos educativos abiertos [SWD(2013) 341 final]. Bruselas. Recuperado de https://goo.gl/l3RwMz

Dagnino, F. M., Antonaci, A., Ott, M., Lavagnino, E., Bellotti, F., Berta,
R., y De Gloria, A. (2015). The eSG Project: A Blended Learning Model for Teaching Entrepreneurship Through Serious Games. European Conference on Innovation and Entrepreneurship (pp. 147-156). Academic Conferences International Limited. Recuperado de https://goo.gl/6WvNkm

De-Marcos, L., Domínguez, A., Saenz-deNavarrete, J., y Pagés, C. (2014). An empirical study comparing gamification and social networking on e-learning. Computers \& Education, 75, 8291. doi: https://doi.org/10.1016/j. compedu.2014.01.012

De-Marcos, L., García-Lopez, E., y GarcíaCabot, A. (2016). On the effectiveness of game-like and social approaches in learning: Comparing educational gaming, gamification \& social networking. Computers \& Education, 95, 99113. doi: https://doi.org/10.1016/j. compedu.2015.12.008

Deterding, S., Dixon, D., Khaled, R., y Nacke, L. (2011). From Game Design Elements to Gamefulness: Defining Gamification. En 15th International Academic MindTrek Conference: Envisioning Future Media Environments (9-15). ACM. Recuperado de https://goo.gl/2n6hOd

Dichev, C., y Dicheva, D. (2013). Support for independent learning in evolving computer science disciplines. Information and Communication Technologies (WICT), 2013 Third World Congress (pp. 202-207). IEEE. doi: https://doi. org/10.1109/wict.2013.7113135

Dicheva, D., Dichev, C., Agre, G., y Angelova, G. (2015). Gamification in education: A systematic mapping study. Educational Technology \& Society, 18(3), 75-88. Recuperado de https://goo.gl/nrFfjF

Drace, K. (2013). Gamification of the laboratory experience to encourage student engagement. $J$ Microbiol Biol Educ, 14(2), 273-274. doi: https://doi. org/10.1128/jmbe.v14i2.632 
Driscoll, M. (2002). Blended learning: Let's get beyond the hype. New York: IBM Global Services

Drott, M. C. (1995). Reexamining the role of conference papers in scholarly communication. Journal of the American Society for Information Science, 46(4), 299. doi: https://doi.org/10.1002/ (sici)1097-4571(199505)46:4<299::aidasi6>3.0.c0;2-0

Faghihi, U., Brautigam, A., Jorgenson, K., Martin, D., Brown, A., Measures, E., y Maldonado-Bouchard, S. (2014). How Gamification Applies for Educational Purpose Specially with College Algebra. Procedia Computer Science, 41, 182187.doi: https://doi.org/10.1016/j. procs.2014.11.102

Ferzli, M., Pigford, K., y Black B. (2015). Development of a gamified learning object (GLO) with Correlated Classroom Activities to Enhance Student Understanding of Evolution. Proceedings ICERI2O15 (pp.2898-2906). IATED. Recuperado de https://goo.gl/PvdelE

Figueiredo, M., Godejord, B., y Rodrigues, J. (2016). The Development of an Interactive Mathematics App for Mobile Learning. 12th International Conference Mobile Learning (pp.75-81). Recuperado de http://toc.proceedings. com/30525webtoc.pdf

Flores, J. F. F. (2015). Using Gamification to Enhance Second Language Learning. Digital Education Review, 27, 32-54. Recuperado de https://goo.gl/iCngS4

Friess, R., Blessing, A., Winter, J., y Zöckler, M. (2015). Film Education for Primary-School Pupils: Gamification and Interactive Storytelling as an Educational Approach to Raise Awareness of Design Structures in Feature Films. European Conference on Games Based Learning (pp. 780-784). Academic Conferences International Limited. Recuperado de https://goo.gl/GdvQwt
Garrison, D. R., y Kanuka, H. (2004). Blended learning: Uncovering its transformative potential in higher education. The internet and higher education, $7(2)$, 95-105. doi: https://doi.org/10.1016/j. heduc.2004.02.001

Graham, C. R. (2013). Emerging practice and research in blended learning. En M. G. Moore, (Ed.), Handbook of distance education. (333-350). Nueva York: Routledge.

Hamari, J., Koivisto, J., y Sarsa, H. (2014). Does gamification work? A literature review of empirical studies on gamification. System Sciences (HICSS), 2014 47th Hawaii International Conference (pp. 3025-3034). IEEE. doi: https://doi.org/10.1109/hicss.2014.377

Hanus, M. D., y Fox, J. (2015). Assessing the effects of gamification in the classroom: A longitudinal study on intrinsic motivation, social comparison, satisfaction, effort, and academic performance. Computers \& Education, 80, 152-161.doi: https://doi. org/10.1016/j.compedu.2014.08.019

Hew, K. F., Huang, B., Chu, K. W. S., y Chiu, D. K. (2016). Engaging Asian students through game mechanics: Findings from two experiment studies. Computers \& Education, 92, 221-236. doi: https://doi. org/10.1016/j.compedu.2015.10.010

Huang, L. Y. (2016). Meaningful Gamification for Journalism Students to Enhance Their Critical Thinking Skills. 1oth European Conference on Games Based Learning: ECGBL 2016 (pp. 289-295). IEEE. Recuperado de https://goo.gl/upm173

Hung, H. T. (2014). Flipping the classroom for English language learners to foster active learning. Computer Assisted Lanquage Learning, 28(1), 81-96. doi: https://doi.org/10.1080/09588221.2014 967701

Iosup, A., y Epema, D. (2014,). An experience report on using gamification in technical higher education. En 45th ACM technical symposium on Computer science 
education (pp. 27-32). ACM. Recuperado de https://goo.gl/ISLuL6

Kapp, K. M. (2012). The gamification of learning and instruction: game-based methods and strategies for training and education. Nueva Jersey: John Wiley \& Sons.

Knutas, A., Ikonen, J., Nikula, U., y Porras, J. (2014). Increasing collaborative communications in a programming course with gamification: a case study. Proceedings of the 15th International Conference on Computer Systems and Technologies (pp. 370-377). ACM. doi: https://doi. org/10.1145/2659532.2659620

Landers, R. N. (2014). Developing a theory of gamified learning: Linking serious games and gamification of learning. Simulation \& Gaming, 45(6), 752-768. doi: https:/ doi.org/10.1177/1046878114563660

Landers, R. N., y Callan, R. C. (2011). Casual social games as serious games: The psychology of gamification in undergraduate education and employee training. Serious games and edutainment applications (pp. 399-423). Londres: Springer London.

Laskowski, M. (2015). Implementing gamification techniques into university study path-A case study. Global Engineering Education Conference (EDUCON), (pp. 582-586). IEEE. doi: $\quad$ https://doi.org/10.1109 educon.2015.7096028

Lemus, B. A., y Pinto, R. (2014). Designing, building and preliminary results of "Cerebrex", a serious educational videogame. Proceedings Conference and Trends (InPACT) (pp. 1-5). Recuperado de https://goo.gl/MJ1XSj

Lemus, B.A., Baessa, Y., y García.J. (2014). Applying the Serious Educational Videogame: Cerebrex to 6th graders for an Educational and Motivational Boost. Proceedings Conference and Trends
(InPACT) (pp.81-85). Recuperado de https://goo.gl/TkgzAR

Lennon, A., Abbott, M., y McIntosh, K. (2015). Chasing higher solar cell efficiencies: Engaging students in learning how solar cells are manufactured. Teaching, Assessment, and Learning for Engineering (TALE), 2015 IEEE International Conference (pp. 267-271). IEEE. doi: https://doi.org/10.1109 tale.2015.7386056

Lim, D. H., Morris, M. L., y Kupritz, V. W. (2007). Online vs. blended learning: Differences in instructional outcomes and learner satisfaction. Journal of Asynchronous Learning Networks, 11(2), 27-42. Recuperado de https://goo.gl DLsgof

López-Pérez, M. V., Pérez-López, M. C., y Rodríguez-Ariza, L. (2011). Blended learning in higher education: Students' perceptions and their relation to outcomes. Computers \& Education, 56(3), 818-826. doi: https://doi.org/10.1016/i. compedu.2010.10.023

Lim, D. H., y Morris, M. L. (2009). Learner and instructional factors influencing learning outcomes within a blended learning environment. Educational Technology \& Society, 12(4), 282-293. Recuperado de https://goo.gl/IJqhK1

Lückemeyer, G. (2015). Virtual blended learning enriched by gamification and social aspects in programming education. Computer Science \& Education (ICCSE), 2015 1oth International Conference on (pp. 438-444). IEEE. doi: https://doi. org/10.1109/iccse.2015.7250286

Martínez-Nicolás, M. M., y SaperasLapiedra, E. (2011). La investigación sobre Comunicación en España (19982007). Análisis de los artículos publicados en revistas científicas. Revista latina de comunicación social, 66, 101-129.doi: https://doi.org/10.4185/rlcs-66-2011226-101-129 
Means, B., Toyama, Y., Murphy, R., y Baki, M. (2013). The effectiveness of online and blended learning: A meta-analysis of the empirical literature. Teachers College Record, 115(3), 1-47. Recuperado de https://goo.gl/R6zred

Mekler, E. D., Bopp, J. A., Tuch, A. N., y Opwis, K. (2014). A systematic review of quantitative studies on the enjoyment of digital entertainment games. Proceedings of the 32nd annual ACM conference on Human factors in computing systems (pp. 927-936). ACM. doi: https://doi. org/10.1145/2556288.2557078

Moskal, P., Dziuban, C., y Hartman, J. (2013). Blended learning: A dangerous idea? The Internet and Higher Education, 18, 15-23. doi: https://doi.org/10.1016/j. iheduc.2012.12.001

Mozelius, P., Collin, J., y Olsson, M. (2015). Visualisation and gamification of e-Learning-Attitudes among course participants. 1oth International Conference on e-Learning ICEL2O15, College of the Bahamas, Nassau, (pp. 227234). Academic Conferences Publishing. Recuperado de https://goo.gl/L9zdd6

Muntean, C. I. (2011). Raising engagement in e-learning through gamification. Proccedings 6th International Conference on Virtual Learning ICVL (pp. 323-329). University of Bucharest Press. Recuperado de https://goo.gl/TN93dA

Nevin, C. R., Westfall, A. O., Rodríguez, J. M., Dempsey, D. M., Cherrington, A., Roy, B., Patel, M., y Willig, J. H., (2014). Gamification as a tool for enhancing graduate medical education. Postgraduate medical journal, 90(1070), 685-693. doi: https://doi.org/10.1136 postgradmedj-2013-132486

Nicholson, S. (2012). A user-centered theoretical framework for meaningful gamification. Games + Learning + Society, 8(1), 1-7. Recuperado de https://goo.gl/ rx32t'
Noor, A. (2013). Envisioning engineering education and practice in the coming intelligence convergence era-a complex adaptive systems approach. Open Enqineering, 3(4), 606-619. doi: https:// doi.org/10.2478/s13531-013-0122-g

Olsson, M., y Mozelius, P. (2016). On design of online learning environments for programming education. Academic Conferences and Publishing International ECEL (pp. 12-24). DIVA. Recuperado de https://goo.gl/6Nlw65

Orooji, F., Taghiyareh, F., y Nasirifard, P. (2015). DoosMooc: An Online Learning Environment Equipped with Innovative Social Interactions. Bulletin of the IEEE Technical Committee on Learning Technology, 17(3), 18. Recuperado de https://goo.gl/p4SEdB

Pedersen, M. K., Svenningsen, A., Dohn, N. B., Lieberoth, A., y Sherson, J. (2016). DiffGame: Game-based mathematics learning for physics. Procedia-Social and Behavioral Sciences, 228, 316322. doi: https://doi.org/10.1016/j. sbspro.2016.07.047

Perez-Sanagustin, M., Hernández-Leo, D., Santos, P., Kloos, C. D., y Blat, J. (2014). Augmenting reality and formality of informal and non-formal settings to enhance blended learning. IEEE Transactions on Learning Technologies, 7(2), 118-131.doi: https:/ doi.org/10.1109/tlt.2014.2312719

Picciano, A. G., Dziuban, C. D., y Graham, C. R. (2013). Blended learning: Research perspectives. New York: Routledge.

Pirker, J., Gutl, C., y Astatke, Y. (2015). Enhancing online and mobile experimentations using gamification strategies. Experiment@ International Conference (pp. 224-229). IEEE. doi: https://doi.org/10.1109/ expat.2015.7463270

Priego, R. G., y Peralta, A. G. (2013). Engagement factors and motivation in e-Learning and blended-learning projects. 
Proceedings of the First International Conference on Technological Ecosystem for Enhancing Multiculturality (pp. 453-460). ACM. doi: https://doi. org/10.1145/2536536.2536606

Priego, R. G., y Peralta, A. G. (2016). How could quality, motivation and engagement be improved in eLearning? Campus Virtuales, 5(1), 74-82. Recuperado de https://goo.gl/5fdgDj

Poon, J. (2013). Blended learning: An institutional approach for enhancing students' learning experiences. Jounal of online learning and teaching and teaching. 9(2), 271. Recuperado de https://goo.gl/ Ysaq8T

Railean, E. A. (2017). Impacts of Digital Revolution on Learning. En User Interface Design of Digital Textbooks (pp. 1-22). Singapur: Springer Singapore. doi:https://doi.org/10.1007/978-981-102456-6 1

Rivera, G. (2004). Modelo de diseño instruccional para programas educativos a distancia. Perfiles educativos, 26(104), 23-114. Recuperado de https://goo.gl/ ym1ZTH

Rodgers, W. M., Hall, C. R., Duncan, L. R., Pearson, E., y Milne, M. I. (2010). Becoming a regular exerciser: Examining change in behavioural regulations among exercise initiates. Psychology of Sport and Exercise, 11(5), 378-386. doi: https://doi. org/10.1016/j.psychsport.2010.04.007

Rovai, A. P., y Jordan, H. (2004). Blended learning and sense of community: A comparative analysis with traditional and fully online graduate courses. The International Review of Research in Open and Distributed Learning, 5(2). doi: https://doi.org/10.19173/irrodl.v5i2.192

Ryan, R. M., y Deci, E. L. (2000). Intrinsic and extrinsic motivations: Classic definitions and new directions. Contemporary educational psychologu, 25(1), 54-67. doi: https://doi.org/10.1006/ceps.1999.1020
Sampieri, R., Collado, C., y Lucio, P. (2014). Metodología de la investigación. Nueva York: Editorial McGraw-Hill.

Simões, J., Redondo, R. D., y Vilas, A. F. (2013). A social gamification framework for a K-6 learning platform. Computers in Human Behavior, 29(2), 345353. doi: https://doi.org/10.1016/i. chb.2012.06.007

Singh, H. (2003). Building effective blended learning programs. Educational Technology, 44(1), 5-27. Recuperado de https://goo.gl/sQI6IJ

Su, C. H., y Cheng, C. H. (2015). A mobile gamification learning system for improving the learning motivation and achievements. Journal of Computer Assisted Learning, 31(3), 268-286. doi: https://doi.org/10.1111/jcal.12088

Tan, M., y Hew, K. F. (2016). Incorporating meaningful gamification in a blended learning research methods class: Examining student learning, engagement, and affective outcomes. Australasian Journal of Educational Technoloqu, 32(5). doi: https://doi.org/10.14742 ajet.2232

Thomas, C., y Berkling, K. (2013). Redesign of a gamified software engineering course. Interactive Collaborative Learning (ICL), 2013 International Conference (pp. 778786). IEEE. doi: https://doi.org/10.1109/ icl.2013.6644707

Velázquez, E. A. P. (2015). The use of bussines simulators in teaching logistics. Interactive Collaborative and Blended Learning (ICBL), 2015 International Conference (pp.57-60).IEEE. doi:https:// doi.org/10.1109/icbl.2015.7387634

Wollscheid, S., Sjaastad, J., y Tømte, C. (2016). The impact of digital devices vs. Pen (cil) and paper on primary school students' writing skills-A research review. Computers \& Education, 95, 19-35. doi: https://doi.org/10.1016/j. compedu.2015.12.001 
Woltering, V., Herrler, A., Spitzer, K., y Spreckelsen, C. (2009). Blended learning positively affects students' satisfaction and the role of the tutor in the problembased learning process: results of a mixed-method evaluation. Advances in Health Sciences Education, 14(5), 725. doi: https://doi.org/10.1007/s10459009-9154-6

Zacharis, N. Z. (2015). A multivariate approach to predicting student outcomes in web-enabled blended learning courses. The Internet and Higher Education, 27, 44-53. doi: https://doi.org/10.1016/j. heduc.2015.05.002

\section{PERFIL ACADÉMICO Y PROFESIONAL DE LOS AUTORES}

Ángel Torres-Toukoumidis. Docente y técnico de revistas científicas de la universidad Politécnica Salesiana, Cuenca-Ecuador. Doctorado en el programa de Doctorado Interuniversitario en Comunicación bajo la línea de investigación "Educomunicación y Media Literacy". Licenciado en Estudios Liberales por la Universidad Metropolitana (Venezuela), homologado en Ciencias Políticas por la Universidad Complutense y Máster en Comunicación con Fines Sociales por la Universidad de Valladolid. Sus principales líneas de investigación son media literacy, gamificación, educación ciudadana, experiencia de usuario.

E-mail: atorrest@ups.edu.ed

\section{DIRECCIÓN DEL AUTOR}

Universidad Politécnica Salesiana, Sede Cuenca

Calle Turuhuayco 3-69 y Calle Vieja,

Cuenca. Ecuador

Luis Miguel Romero Rodríguez. Docente de la Universidad Internacional de la Rioja y Profesor Invitado de la Universidad Internacional de Andalucía. Doctorado en el programa Interuniversitario en Comunicación bajo la línea de investigación "Educomunicación y Media Literacy". Investigador de la Red Interuniversitaria Euroamericana sobre Competencias Mediáticas para la Ciudadanía Alfamed y del Grupo Ágora de la Universidad de Huelva. Sus principales líneas de investigación son media literacy, epistemología y pragmática de la desinformación y sus estratagemas. E-mail: huismiguel.romero@unir.net

\section{DIRECCIÓN DEL AUTOR}

Universidad Internacional de La Rioja, España

Calle Mackay Macdonald, $\mathrm{N}^{\circ} 4$, piso 4, puerta 4-D.

Huelva, 21004 
Amor Pérez Rodríguez. Profesora Titular de Universidad en el Departamento de Filología de la Universidad de Huelva. Investigadora de la Red Internacional Alfamed y del Grupo Ágora de la Universidad de Huelva. Sus líneas de investigación se centran en el desarrollo de la competencia mediática y alfabetización mediática, nuevos lenguajes de la comunicación y didáctica de la lengua y la literatura.

E-mail: amor@uhu.es

\section{DIRECCIÓN DE LA AUTORA}

Facultad de Humanidades

Campus de «El Carmen»

Avda. de las Fuerzas Armadas, S/N. 21007 Huelva

Fecha de recepción del artículo: 12/04/2017

Fecha de aceptación del artículo: 10/05/2017

\section{Como citar este artículo:}

Torres-Toukoumidis, A., Romero-Rodríguez, L. M., y Pérez-Rodríguez, A. M. (2018). Ludificación y sus posibilidades en el entorno de blended learning: revisión documental. RIED. Revista Iberoamericana de Educación a Distancia, 21(1), pp. 95-111. doi: http://dx.doi.org/10.5944/ried.21.1.18792 